più alta nelle prostitute $(6.3 \%)$ rispetto alle altre due popolazioni (autoctone $1.9 \%$, immigrate non prostitute $2.8 \%$ ) a conferma di una trasmissione per via sessuale. Per quanto concerne i micoplasmi, anche qui assistiamo ad un progressivo aumento nelle tre popolazioni. Essi infatti passano dal $24.8 \%$ della popolazione autoctona al $28.4 \%$ delle donne immigrate non prostitute, sino ad arrivare al $37.7 \%$ nelle prostitute.

Conclusioni La crescente percentuale di isolamento di microrganismi patogeni riscontrata nelle pazienti immigrate rispetto alla popolazione autoctona conferma che lo scarso livello di conoscenza delle principali MST e loro modalità di trasmissione, il basso livello di scolarizzazione, le errate abitudini igieniche rappresentano fattori di rischio per l'insorgenza di tali patologie. Queste ultime risultano ancora più elevate nelle prostitute a causa della promiscuità e dell'elevato numero di partner occasionali, nonché dello scarso utilizzo del condom.

\title{
LE INFEZIONI CERVICO-VAGINALI: CONFRONTO TRA TRE DIVERSE POPOLAZIONI
}

\author{
Cuniato V.', Di Martino M. ', Chianese M.G. ' Natale R. ', \\ D'Isanto R. ${ }^{2}$, Nocera E ${ }^{3}$ Bozzelli L. ${ }^{3}$ Longanella A.. ${ }^{4}$ \\ ' Centro Medico-sociale J. E. Masslo, Castel-volturno, (CE). \\ 2 Ospedale S.M.Delle Grazie Pozzuoli (NA). \\ ${ }^{3}$ Centro Diagnostico S.Ciro, Portici (NA). \\ ${ }^{4}$ Laboratorio "Diagnostica", Castel S.Giorgio(SA).
}

Introduzione Le infezioni cervico-vaginali rappresentano una patologia di frequente riscontro nell'ambito della popolazione femminile in età fertile ed assumono una particolare rilevanza clinica ed epidemiologica nelle prostitute soprattutto se provenienti dai paesi in via di sviluppo.

Obiettivi Al fine di valutare la frequenza di infezioni cervico-vaginali è stato condotto, nel periodo compreso tra marzo 2004 e marzo 2005, uno studio prospettico su 3 popolazioni di pazienti sintomatiche: donne autoctone (350), donne immigrate extracomunitarie (209), donne immigrate extracomunitarie dedite alla prostituzione (305).

Materiali e metodi. Sede vaginale: un tampone per l'esame microscopico sia a fresco sia dopo colorazione di Gram; ed uno per l'esame colturale mediante semina sui terreni:agar Sangue Columbia, agar Columbia CNA, Mac Conkey, agar Sabouraud addizionato con cloramfenicolo, agar cioccolato (piastre BD). Sede cervicale: un tampone per la ricerca di Mycoplasma hominis e Ureaplasma urealyticum, eseguita utilizzando il Kit Mycoplasma IST (bioMérieux).

Risultati Candida albicans rappresenta l'agente etiologico di più frequente riscontro nelle 3 popolazioni in esame anche se in diverse proporzioni.

Seguono Gardnerella vaginalis, Streptococcus. agalactiae, Enterobacteriaceae, Staphylococcus aureus Trichomonas vaginalis presenta una percentuale di isolamento nettamente 